\title{
A Study of the Social Perception of Technology in Public Spaces in Alajuela Central Canton, Costa Rica
}

\author{
Andrés Víquez Víquez \\ Escuela de Ingeniería en Computación \\ Instituto Tecnológico de Costa Rica \\ Alajuela, Costa Rica \\ anviquez@itcr.ac.cr
}

\author{
Irene Hernández Ruiz \\ Escuela de Informática \\ Universidad Nacional de Costa Rica \\ Heredia, Costa Rica \\ irene.hernandez.ruiz@una.cr
}

\author{
Fabián Pérez Gutiérrez \\ Asociación Costarricense de Ingenieros en \\ Construcción \\ Colegio Federado de Ingenieros y \\ Arquitectos \\ San José, Costa Rica \\ fperezg@cfia.or.cr
}

\begin{abstract}
The present work makes an analysis of the results of a study of the social perception of technology applied in the public spaces of the central canton of Alajuela, Costa Rica. This study is of a non-probabilistic type, commonly used in exploratory research, which counted with the participation of 122 visitors, 55 women and 67 men, aged between 15 and 79 years, in 6 parks of the central canton of Alajuela, using QGIS tool for the spatial analysis of the data.
\end{abstract}

Keywords - Citizenship, Culture, Technology, QGIS, Public Space.

\section{INTRODUCCIÓN}

Paramo [1] entiende el espacio público "como el escenario donde se condensa la vida urbana", mientras que Licona [2] lo entiende "cómo ordenador de las prácticas colectivas e individuales que expresa procesos de apropiación y se entrelaza con el modo de vida de las personas y sus diferentes formas de interacción social, mediante las cuales se exhiben las prácticas sociales que logran identificar y cohesionar los grupos culturales".

En ese sentido, algunas ciudades de América han desarrollado proyectos para fomentar el uso del espacio público, como es el caso de la ciudad de Panamá, la cual cuenta desde el 2017 con 50 puntos de acceso gratuito a internet inalámbrico, incluidas decenas de paradas de autobús a las que se han incorporado pantallas digitales con información sobre la urbe. La Alcaldía de Panamá, promotora de la iniciativa, indicó que el servicio de conectividad Smart City pone a la ciudad a la vanguardia de la tecnología y ayuda a prestar una mejor calidad de internet por la colocación de "small cells", pequeñas antenas de amplificación de señal del servicio celular [3].

Otro caso es el de la ciudad de Nueva York, que dedica cada vez más zonas asfaltadas para la ampliación de senderos y espacios abiertos, o la ciudad de Melbourne, que ha modificado el uso de 86 hectáreas de calles y otros espacios infrautilizados en los últimos 30 años, y ha adaptado nuevo espacio residencial de media a alta densidad alrededor de sus estaciones ferroviarias y redes de transporte público por carretera [4].El Índice IESE Cities in Motion se ha diseñado con el objetivo de construir un indicador «superador» - en cuanto a su completitud, sus propiedades, su comparabilidad, su calidad y la objetividad de la información incluida - que permita medir la sostenibilidad de cara al futuro de las principales ciudades del mundo, al igual que la calidad de vida de sus habitantes. Para el año 2018, las primeras posiciones las ocuparon: en primer lugar, Nueva YorkEstados Unidos con un índice de 100.00; en segundo lugar, Londres-Reino Unido con 99.27; París-Francia en tercer lugar con 90.20 y en cuarto lugar Tokio-Japón con 84.38 [5].

Por su parte, Costa Rica cuenta actualmente con el Plan Nacional de Ordenamiento Territorial (PLANOT) [6], establecido a ejecutarse desde el 2014 al 2020, que se constituye como el documento de gestión operativa que establece las acciones estratégicas en respuesta a las metas definidas en la Política Nacional de Ordenamiento Territorial (PNOT) 20122040, oficializada mediante el Decreto del Poder Ejecutivo $\mathrm{N}^{\circ}$ 37623 PLAN-MINAET-MIVAH, publicado en el diario oficial La Gaceta del 10 de mayo del 2013. El PLANOT posee tres ejes estructurales para llevarlo a cabo, los cuales son: la calidad del hábitat, la competitividad territorial y la protección y manejo ambiental.

No obstante, el constante crecimiento de la virtualidad de las relaciones sociales, ha producido múltiples transformaciones en la forma de interactuar con y en los espacios urbanos y como consecuencia, en su factor cuantitativo de los espacios públicos. Lefebvre [7] ya advertía que la relación del espacio con la sociedad proviene de varias ciencias, siendo una de ellas la tecnología. En este aspecto, la ciudad de Alajuela en noviembre del 2018 dio un nuevo paso en su decisión de convertirse en una "ciudad inteligente", con la instalación en el parque Juan Santamaría del primero de 20 postes inteligentes que ofrecen servicios de seguridad y comunicación a la población [8]. La iniciativa es gracias al convenio establecido con el Instituto Costarricense de Electricidad (ICE) y Radiográfica Costarricense (RACSA).

Este estudio se enmarca en el apartado de paisajes, áreas verdes, recreativas y espacios públicos, específicamente los parques municipales del casco central de la ciudad de Alajuela. Para este trabajo se presentan los siguientes apartados: metodología, lugar y participantes del estudio, instrumento aplicado, herramientas utilizadas, resultados del estudio, conclusiones y trabajo futuro. 


\section{MetodologíA}

Como metodología de trabajo para la recolección y procesamiento de datos se propuso un estudio no probabilístico, comúnmente utilizados en investigaciones exploratorias, donde los sujetos de estudio serán seleccionados siguiendo determinados criterios procurando, en la medida de lo posible, que la muestra sea representativa. Para esto se realizará un muestreo discrecional, donde basado en el conocimiento y juicio de los profesionales inmersos en el proyecto, se seleccionarán los individuos más "representativos" o "adecuados" para los fines del estudio. Las entrevistas fueron realizadas cara a cara a un total de 122 visitantes de los parques, que tuvieron una duración máxima de 5 minutos y fueron apoyadas con el uso de dispositivos móviles y una herramienta CAPI (entrevistas asistidas por computadora por sus siglas en inglés) de manera que la digitalización e integración de datos fue llevada a cabo en tiempo real.

\section{PARTICIPANTES Y LUGAR DE ESTUDIO}

Los participantes del estudio fueron:

- Entrevistadores: el profesor y 13 estudiantes del curso Administración de Proyectos. Como parte de la evaluación del curso, los estudiantes debían desarrollar un proyecto grupal para el desarrollo de un estudio del comportamiento de la población en la temática de las TICs. Este curso pertenece al tercer año y se imparte cada semestre.

- Entrevistados: personas que se encuentran en la ciudad de Alajuela, los cuales pueden ser visitantes (regulares o no regulares) y vecinos.

La delimitación de este estudio fue el casco central del cantón de Alajuela. De esta manera los parques seleccionados fueron: Parque Central, Parque Juan Santamaría, Parque el Arroyo, Parque Eloy Alfaro (conocido como Parque de Palmares), Parque Próspero Fernández (conocido como Parque del Cementerio) y Parque Calián Vargas; que fueron seleccionados por tener una mayor afluencia de visitantes.

En la figura 1, se presenta el espacio geográfico donde se desarrolló el estudio.

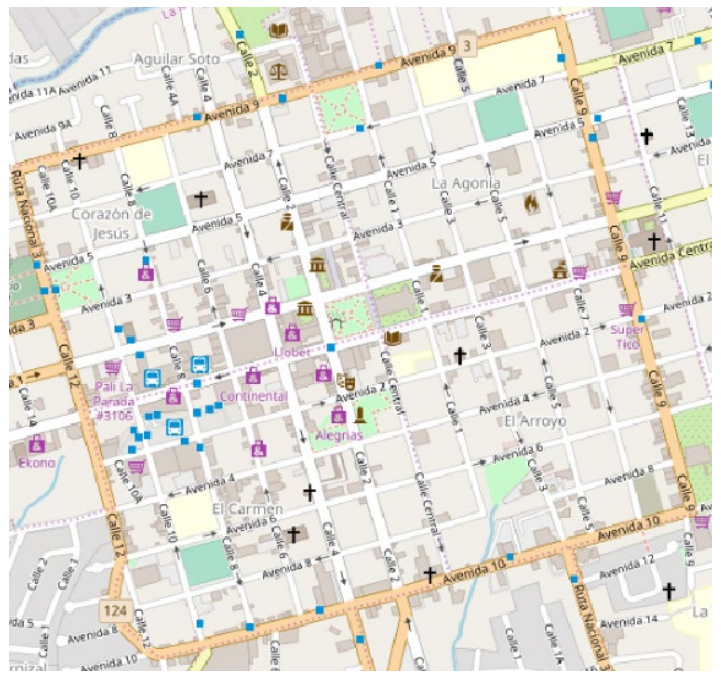

Figura 1. Espacio geográfico donde se desarrolló el estudio
FUENTE: OPEN STREeT MAPS

\section{INSTRUMENTO APLICADO}

En el cuadro 1, se presenta el instrumento aplicado a los visitantes de los parques:

1. Edad

2. Sexo: Masculino Femenino

3. ¿Qué días de la semana visita usted el parque?

- $\quad$ Entre semana.

- $\quad$ Fines de semana.

- Cualquier día de la semana.

4. ¿Qué tan frecuentemente utiliza usted este parque?

- 1.A menudo (3 o más veces por semana)

- 2.1-2 veces por semana

- 3.1-2 veces por mes

- 4.En ocasiones (más de 3 veces por año)

- 5.Rara vez (1 o 2 veces por año o menos)

5. ¿Con quién suele visitar el parque?

- $\quad$ 1.Solo (a)

- 2.Familiares

- 3.Amigos

- 4.Pareja

- 5.Mascotas

- $\quad$ NS / NR

6. ¿Cuál es la principal razón por la que usted visita el parque?

- Traer a los niños a jugar

- Cuidar y acompañar a un adulto mayor

- Sacar a la mascota a pasear

- Salir a hacer deporte

- Para estudiar

- Para tener un espacio de recreación

- Para estar con su pareja

- Hacer compras

- $\quad$ Porque queda de camino a mi casa o trabajo

- Otro:

- $\quad \mathrm{NS} / \mathrm{NR}$

7. ¿Considera que este parque está adecuadamente equipado?

- 1. Totalmente desacuerdo - 2. En desacuerdo - 3. Neutral 4. De acuerdo - 5. Totalmente de acuerdo - 6. NS / NR

8. ¿Considera que el uso de pantallas interactivas (informativas) podrían ser de utilidad para los visitantes del parque? Sí-No

9. ¿Qué información consideraría relevante que muestren las pantallas interactivas (informativas) a los visitantes del parque?

- Mapas turísticos de la ciudad

- Comercios

- Paradas de autobuses

- Restaurantes y bares

- Hoteles y hosteles

- Instituciones públicas

- Integración con aplicaciones populares: Trip Advisor, Google Maps

- Otro:

10. ¿Instalaría en su celular una aplicación que le permitiera acceder al contenido de las pantallas interactivas (informativas)? Sí-NoNo aplica

11. ¿Utiliza el servicio de internet inalámbrico (WI-FI) del parque? Sí-No-No aplica

12. ¿Por qué razón no utiliza el servicio de internet inalámbrica WIFI del parque?

- Temor a ser asaltado.

- La conexión es lenta.

- Desconfianza de la seguridad de la conexión.

- Nunca funciona.

- $\quad$ No utilizo dispositivos que se conecten a Internet.

- No sabía que había WI-FI. 
- Otro

13. ¿Considera el parque un espacio seguro? Sí-No

14. ¿Considera que las cámaras de video vigilancia son necesarias en el parque? Sí-No

15. ¿Considera que el parque debería contar con dispositivos electrónicos que permitan a los visitantes alertar situaciones de emergencia o peligro? Sí-No

16. ¿Considera importante la instalación de centros de carga de dispositivos para los visitantes del parque? Sí-No

17. ¿Está de acuerdo con la siguiente declaración: "este parque satisface sus necesidades recreativas"?

- Totalmente desacuerdo

- Levemente en desacuerdo

- Neutral

- Levemente de acuerdo

- Muy de acuerdo

18. ¿Cuál considera que es el principal problema que afecta este parque?

- Contaminación

- Delincuencia.

- Ruido excesivo.

- $\quad$ Falta de mantenimiento.

- Propagación de enfermedades.

- Indigencia.

- Prostitución.

- Otro:

- $\quad \mathrm{NS} / \mathrm{NR}$

19. ¿Qué cree usted que necesita mejorar el parque como prioridad?

- No necesitan mejorar.

- Programas (ej. conciertos en el parque, arte en el parque, eventos especiales $u$ otros eventos organizados)

- Políticas de permisos (ej. para el uso de campos deportivos eventos especiales, alquiler de las instalaciones del parque, etc.)

- $\quad$ Proteger los espacios verdes del desarrollo urbano.

- Mantenimiento (ej. mantenimiento general del parque, edificios, árboles, jardines y limpieza de basura)

- $\quad$ Parques infantiles (columpios, toboganes, etc.)

- La seguridad.

- Facilidades tecnológicas (ej. WI-FI, centros de carga, pantallas interactivas, etc.)

20. ¿Cuál es su situación actual? Trabaja-Estudia-Está desempleadoNo trabaja-NS / NR

21. ¿Cuál es su último año de estudios aprobado? Sin escolaridad, Primaria incompleta, Primaria completa Secundaria incompleta, Secundaria completa, Educación técnica, Universitaria incompleta, Universitaria completa.

22. ¿Dónde vive la persona (según barrio)?

\section{FUENTE: ELABORACIÓN PROPIA}

\section{HERRAMIENTAS TIC UTILIZADAS}

Para llevar a cabo esta investigación se utilizaron las siguientes herramientas TIC:

- GIS Cloud: utilizando esta herramienta los estudiantes del curso desde sus teléfonos móviles ingresaron los datos del cuestionario realizado y registran la información de los diferentes puntos de los parques donde realizaron las entrevistas. Posteriormente, los datos recolectados se exportaron a un formato Shapefile para ser analizados en la herramienta QGIS [9].

- QGIS: es un sistema de información geográfica de código libre [10] para plataformas GNU/Linux, Unix, Mac OS, Microsoft Windows y Android, para la creación y gestión de la información geográfica. Utilizando este software los investigadores pudieron analizar los resultados, elaborar los mapas de la investigación, generar conclusiones y trabajo a futuro.

\section{RESUlTADOS DEL ESTUDiO}

La muestra del estudio está compuesta por 122 entrevistas a visitantes de los parques que se describe seguidamente:

\begin{tabular}{|l|c|c|c|c|c|}
\hline \multirow{2}{*}{ Género } & \multicolumn{5}{|c|}{ Edad } \\
\cline { 2 - 6 } & $15-21$ & $22-37$ & $38-53$ & $54-72$ & $73-90$ \\
\hline Femenino & 14 & 26 & 11 & 4 & 0 \\
\hline Masculino & 16 & 22 & 13 & 15 & 1 \\
\hline Total & $\mathbf{3 0}$ & $\mathbf{4 8}$ & $\mathbf{2 4}$ & $\mathbf{1 9}$ & $\mathbf{1}$ \\
\hline
\end{tabular}

TABLA 1. DESCRIPCIÓN DE LOS ENTREVISTADOS POR GÉNERO Y EDAD. FUENTE: ELABORACIÓN PROPIA

En la figura 2 que se muestra a continuación se pueden observar las concentraciones de entrevistas, donde entre más oscuro sea el color, mayor será cantidad de entrevistas aplicadas.

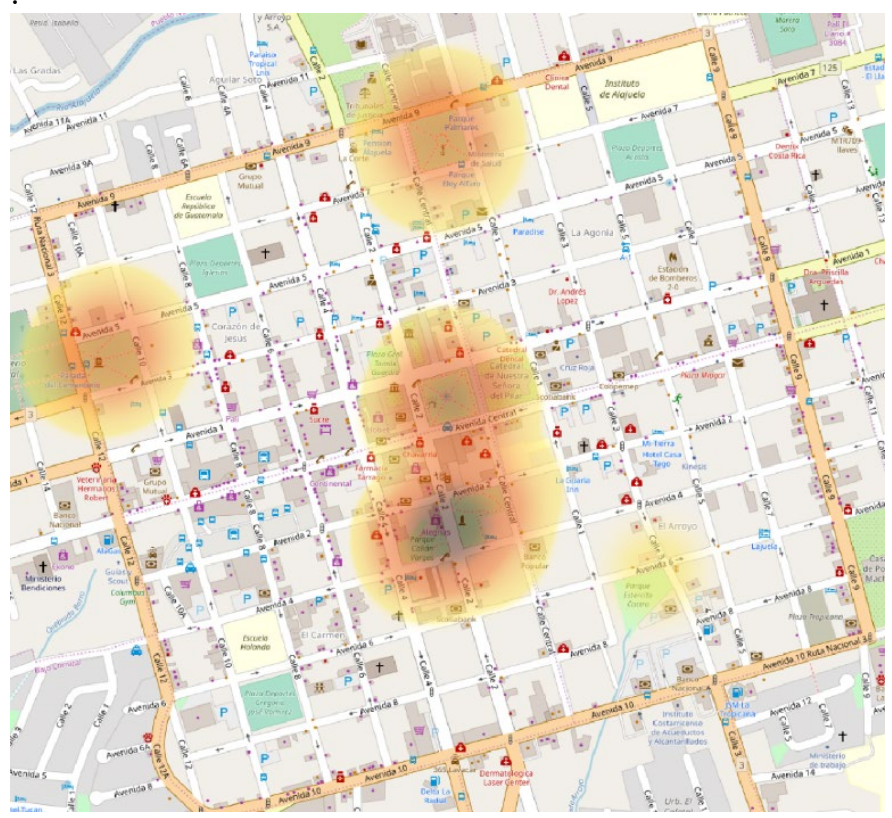

FIGURA 2. MAPA DE CONCENTRACIONES DE ENTREVISTAS FUENTE: ELABORACIÓN PROPIA

Seguidamente se proceden a resumir los principales hallazgos encontrados en el estudio:

A. Cruce entre la frecuencia de uso y la percepción de adecuado equipamiento del parque por los visitantes

\begin{tabular}{|l|c|c|c|c|c|}
\hline $\begin{array}{l}\text { Frecuencia con la que visita el } \\
\text { parque }\end{array}$ & $\mathbf{1}$ & $\mathbf{2}$ & $\mathbf{3}$ & $\mathbf{4}$ & $\mathbf{5}$ \\
\hline $1-2$ veces por mes. & 4 & 5 & 6 & 4 & 4 \\
\hline $1-2$ veces por semana. & 6 & 9 & 8 & 6 & 1 \\
\hline $\begin{array}{l}\text { A menudo (3 o más veces por } \\
\text { semana). }\end{array}$ & 10 & 9 & 3 & 6 & 0 \\
\hline $\begin{array}{l}\text { En ocasiones (más de 3 veces por } \\
\text { año). }\end{array}$ & 2 & 2 & 3 & 2 & 0 \\
\hline $\begin{array}{l}\text { Rara vez (1 o 2 veces por año o } \\
\text { menos). }\end{array}$ & 3 & 8 & 4 & 9 & 7 \\
\hline
\end{tabular}


TABLA 2. CRUCE ENTRE LA FRECUENCIA DE USO Y LA PERCEPCIÓN DE ADECUADO EQUIPAMIENTO DEL PARQUE. FUENTE: ELABORACIÓN PROPIA

De la tabla anterior se desprende que los entrevistados que más frecuentan los parques, son los que consideran que no se encuentra apropiadamente adecuado; mientras que los que menos lo visitan, son los que consideran que sí se encuentran apropiadamente equipados.

\section{B. Razón de uso de los parques por los visitantes}

\begin{tabular}{|l|c|c|c|}
\hline $\begin{array}{l}\text { Razones para visitar los } \\
\text { parques }\end{array}$ & Masculino & Femenino & Total \\
\hline Hacer compras. & 2 & 3 & 5 \\
\hline No sabe / No responde & 2 & 1 & 3 \\
\hline Otro & 5 & 8 & 13 \\
\hline Para estar con su pareja. & 0 & 5 & 7 \\
\hline Para estudiar. & 20 & 27 & 1 \\
\hline $\begin{array}{l}\text { Para tener un espacio de } \\
\text { recreación. }\end{array}$ & 5 & 11 & 16 \\
\hline $\begin{array}{l}\text { Porque queda de camino a mi } \\
\text { casa o trabajo. }\end{array}$ & 0 & 2 & 2 \\
\hline Sacar a la mascota a pasear. & 0 & 1 & 1 \\
\hline Salir a hacer deporte. & 19 & 8 & 27 \\
\hline Traer a los niños a jugar. & $\mathbf{5 5}$ & $\mathbf{6 7}$ & $\mathbf{1 2 2}$ \\
\hline Total & & & \\
\hline
\end{tabular}

TABLA 3. RAZONES POR LA QUE SE VISITAN LOS PARQUES. FUENTE: Elaboración Propia

De la tabla anterior se desprende que las entrevistadas visitan los parques principalmente para tener un espacio de recreación y para traer los niños a jugar; mientras que los entrevistados, los frecuentan igualmente para tener un espacio recreación $\mathrm{y}$ porque queda de camino a la casa o al trabajo.

\section{Pantallas interactivas y aplicación móvil}

¿Considera el uso de pantallas interactivas e informátivas de utilidad para los visitantes del parque?

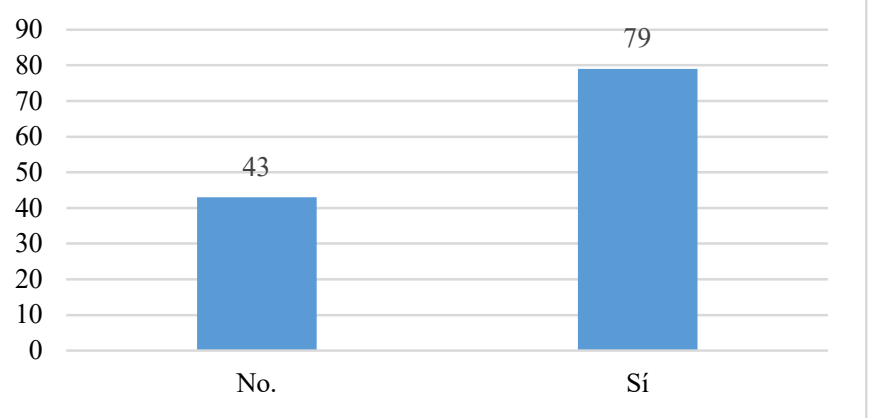

Figura 8. GRÁFICO de OPINIÓN DE UTILIDAd dE PANTALLAS INTERACTIVAS EN LOS PARQUES. FUENTE: ELABORACIÓN PROPIA

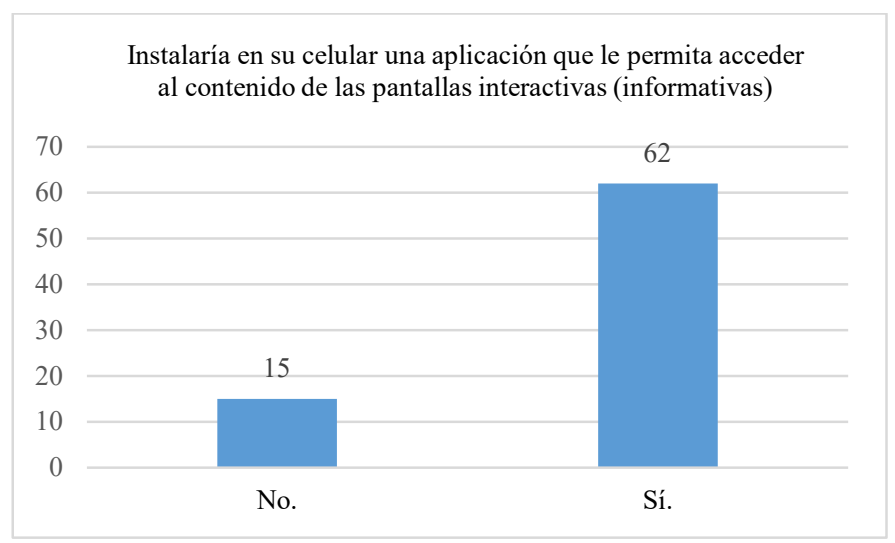

Figura 9. GRÁFico de OPINIÓN PARA INSTALAR UNA APLICACIÓN QUE PERMITA ACCEDER AL CONTENIDO DE LAS PANTALLAS INTERACTIVAS DESDE LOS TELÉFONOS. FUENTE: ELABORACIÓN PROPIA

Cómo podemos apreciar en los gráficos anteriores, la mayoría de los entrevistados consideran de utilidad el uso de pantallas interactivas en los parques y estarían dispuestos a instalar una aplicación que les permita acceder a los contenidos de las pantallas.

\section{Uso del internet inalámbrico en los parques}

¿Utiliza el servicio de internet inalámbrica (WI-FI) del parque?

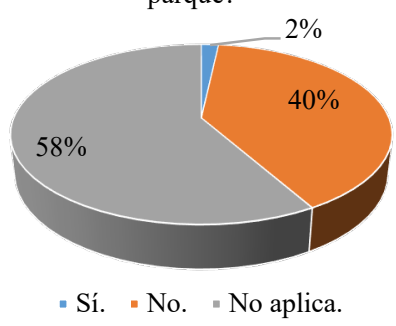

FIGURA 10. GRÁFICO DE SI UTILIZA EL SERVICIO DE INTERNET INALÁMBRICA (WI-FI) DEL PARQUE. FUENTE: ELABORACIÓN PROPIA

Razones para no utilizar el servicio de internet inalámbrica WI-FI del parque

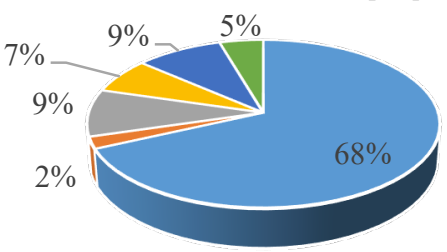

- No sabía que había WI-FI.

- La conexión es lenta.

- No utilizo dispositivos que se conecten a Internet.

- Nunca funciona.

- Otro.

- Temor a ser asaltado

Figura 11. GRÁFICO dE RAZONES DE POR QUÉ NO UTILZA EL SERVICIO DE INTERNET INALÁMBRICA (WI-FI) DEL PARQUE. FUENTE: ELABORACIÓN PROPIA 
Como podemos apreciar en las figuras anteriores, descartando las respuestas de No aplica debido a que son parques donde no existe servicio de Internet Inalámbrica para los visitantes, la gran mayoría de entrevistados no usan el servicio debido a que desconocen de la disponibilidad del mismo.

\section{E. Percepción de seguridad, cámaras de vigilancia y botones de alerta para casos de emergencia o situaciones de riesgo}

Como se aprecia en el mapa de la figura a continuación, la mayoría de entrevistados se sienten seguros cuando transitan o visitan los parques; sin embargo, también manifiestan que no transitarían estos lugares en horas nocturnas. Igualmente manifiestan que para mejorar la convivencia es necesario reforzar la seguridad en los parques a través de la implementación de cámaras de vigilancia en los parques, así como los dispositivos electrónicos para alertar de situaciones de emergencia o peligro.

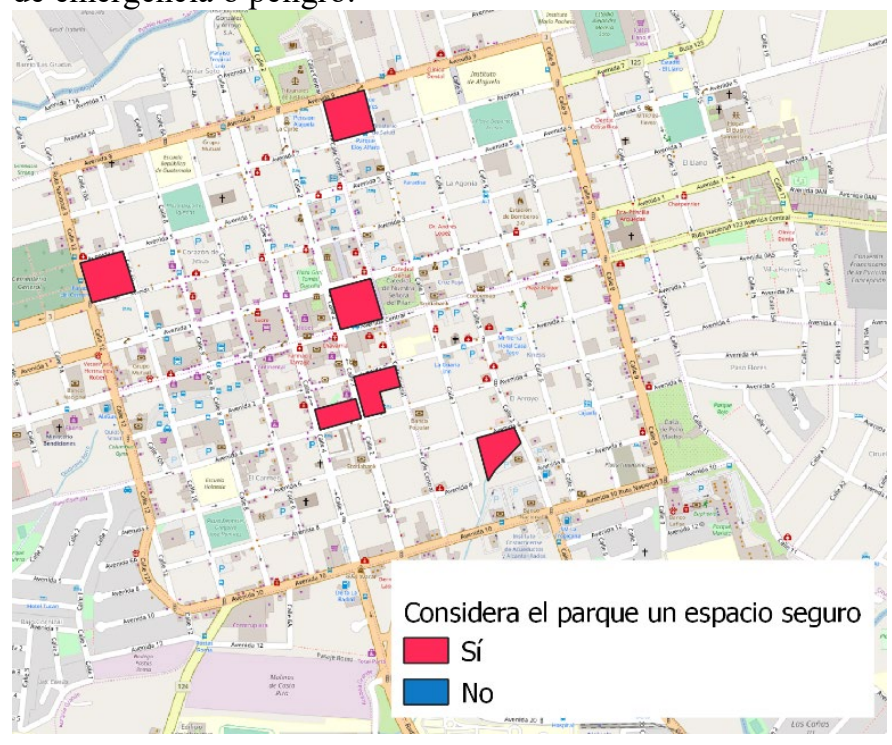

FIGURA 12. MAPA DE PERCEPCIÓN DE SEGURIDAD DE LOS PARQUES POR LOS ENTREVISTADOS. FUENTE: ELABORACIÓN PROPIA

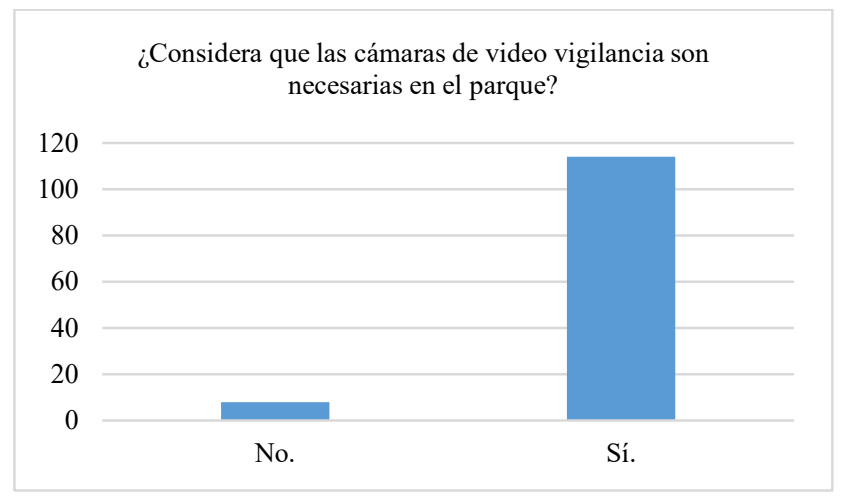

Figura 13. Gráfico de si considera que las cámaras de vigilancia son necesarias en los parques. Fuente: Elaboración propia

Por su parte, en la figura 14 se observa si los entrevistados consideran importante que el parque cuente con dispositivos móviles para alertar situaciones de emergencia o peligro. Para ello se puede encontrar que el $88 \%$ si considera importante este tipo de herramienta.

\section{¿Considera que el parque debería contar con dispositivos electrónicos que permitan a los visitantes alertar situaciones de emergencia o peligro?}

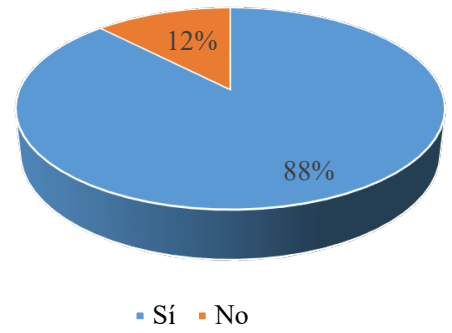

Figura 14. GRÁFICO de SI CONSIDERA QUE LOS DISPOSITIVOS ELECTRÓNICOS QUE PERMITAN ALERTAS DE EMERGENCIAS O PELIGROS SON NECESARIAS EN LOS PARQUES. FUENTE: ELABORACIÓN PROPIA

\section{F. Problemáticas de los parques}

Como se puede apreciar en la figura a continuación, los principales problemas que consideran los entrevistados de los parques son en primera instancia la delincuencia, seguido de la falta de mantenimiento y la indigencia.

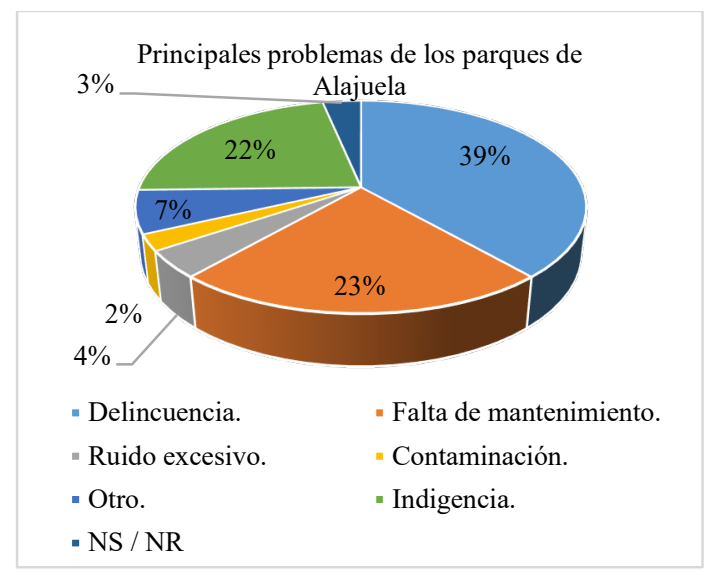

FiguRa 15. GRÁFICO DE PRINCIPALES PROBLEMAS DE LOS PARQUES DE ALAJUELA. FUENTE: ELABORACIÓN PROPIA

Como se puede apreciar en la figura anterior, los principales problemas que consideran los entrevistados de los parques son en primera instancia la delincuencia, seguido de la falta de mantenimiento y la indigencia. A continuación, se muestra un mapa con la percepción de los principales problemas por cada parque.

Seguidamente, se muestra un mapa con la percepción de los principales problemas por cada parque. 


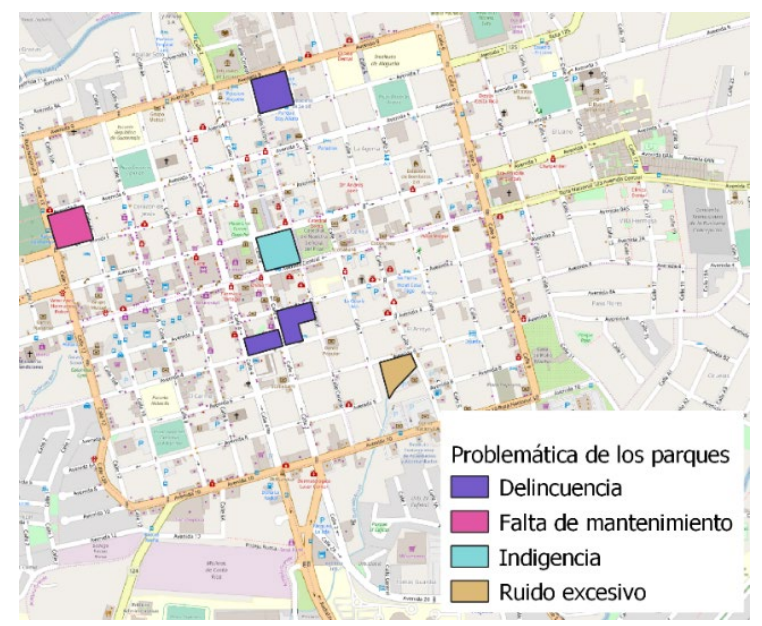

FigURA 16. MAPA DE PERCEPCIÓN DE PROBLEMÁTICAS POR PARQUE. FUENTE: ELABORACIÓN PROPIA

\section{CONCLUSIONES}

- La municipalidad está realizando inversiones en los espacios públicos para convertir a Alajuela en una ciudad digital; sin embargo, la gran mayoría de los entrevistados desconocían de los esfuerzos que se estaban realizando. Esto señala una deficiencia en la efectividad de los medios de comunicación que está utilizando la Municipalidad y una revaloración de los mismos para alcanzar una comunicación efectiva con la población.

- La mayoría de entrevistados tienden a ser personas anuentes a la utilización de a la tecnología, por lo que las inversiones que se realice la Municipalidad para crear una ciudad digital, serían bien aprovechadas por la población.

- La problemática principal que los visitantes perciben en los parques es la delincuencia, por lo que la Municipalidad debe dirigir sus inversiones tecnológicas, en primera instancia, en asegurar que la población se sienta segura en los espacios públicos, antes de realizar otras inversiones más superfluas en tecnología.

- A nivel de resultados, no existen variaciones significativas cuando la variable género es incluida en el análisis. Sin embargo, cuando se distingue la razón de uso del parque y el género del entrevistado, aparece un fenómeno social en los resultados en torno a las dinámicas de convivencia: mientras los hombres utilizan estos sitios porque les quedan de camino a casa o al trabajo, las mujeres lo utilizan para llevar a los niños a jugar.

\section{VIII.TRABAJO A FUTURO}

- Presentar los hallazgos de esta investigación al Consejo Municipal de Alajuela, que permitan ser un insumo en el plan estratégico anual que realiza la Municipalidad.

- Replicar el estudio bajo un modelo probabilístico, que permita obtener resultados con una confianza y margen de error para una adecuada toma de decisiones.

- Reforzar el instrumento con consultas a la población que no sólo permitan conocer cuál es la realidad de la percepción de la aplicación de la tecnología en los espacios públicos del cantón, sino que permitan a las Autoridades desarrollar estrategias participativas y de intervención para alcanzar una equidad de género.

\section{AgRadecimiEnTo}

La investigación presentada en este artículo ha sido posible gracias a la guía del profesor y a la colaboración de los estudiantes del curso de Administración de Proyectos de la carrera de Ingeniería en Computación del Centro Académico de Alajuela.

\section{REFERENCIAS}

[1] Páramo, P. (2004). Algunos conceptos para una perspectiva optimista de vivir la ciudad. Revista Territorios, 10-11, 91-109.

[2] Licona, E. (2007). Habitar y significar la ciudad. Puebla.Conacyt y Casa Abierta al Tiempo.

[3] EFE (2017).Consultado:

https://www.efe.com/efe/america/tecnologia/ciudad-de-panama-instalainternet-gratuito-en-50-espacios-publicos/20000036-3150673

[4]Melbourne (2019),

Consultado

https://www.melbourne.vic.gov.au/community/parks-openspaces/Pages/parks-open-spaces.aspx

[5] IESE Cities in Motion (2018). Consultado en: https://media.iese.edu/research/pdfs/ST-0471.pdf

[6] Plan Nacional de Ordenamiento Territorial (2013). Consultado en: https://www.mivah.go.cr/Documentos/politicas_directrices_planes/pnot/PLA NOT_2013-12-03.pdf

[7] Lefebvre, H. (1974). La producción del espacio. En: Papers: revista de sociología, N. 3, 1974, p. 219-229. ISSN 0210-2862

[8] El Mundo (2018). Consultado en:https://www.elmundo.cr/municipales/iceinstala-en-alajuela-primer-poste-inteligente-en-el-parque-juan-santamaria/

[9] GIS CLOUD (2019). Consultado en: https://www.giscloud.com/

[10] QGIS (2019). Consultado en: https://www.qgis.org/es/site/ 